\title{
Desafíos metodológicos para el estudio de la reflexión en contexto de formación docente
}

\author{
Methodological challenges for the study of the reflection in a teaching training context
}

Desafios metodológicos para o estudo da reflexão no contexto da formação de professores

\author{
Enrique Correa Molina, ${ }^{a}$ Philippe Chaubet, ${ }^{b}$ Simon Collin, ${ }^{c}$ Colette Gervais ${ }^{d}$ \\ aUniversité de Sherbrooke. Correo electrónico: Enrique.correa.molina@usherbrooke.ca \\ bUniversité du Québec à Montréal. Correo electrónico: Chaubet.philippe@uqam.ca \\ cUniversité du Québec à Montréal. Correo electrónico: Collin.simon@uqam.ca \\ dUniversité de Montréal. Correo electrónico: Colette.gervais@umontreal.ca
}

\begin{abstract}
RESUMEN
Este artículo realiza una convergencia de tres de nuestros trabajos sobre el concepto de reflexión y los enfoques metodológicos que hemos privilegiado en ellos. Él constituye la secuencia lógica de otro publicado por el mismo equipo de autores en 2010 presentando una visión crítica de la reflexión. En primer lugar, en este texto, presentamos sumariamente nuestra visión del concepto para, luego, situar nuestras respectivas investigaciones y poner en relieve los desafíos metodológicos que debimos enfrentar para estudiar el proceso de reflexión y el proceso de desarrollo de la reflexión. La descripción de nuestros estudios y el ejercicio de diálogo reflexivo que llevamos a cabo, nos permiten señalar que la observación de la reflexión en contexto de formación inicial necesita el recurso de manifestaciones que pueden ser del orden de la acción o del lenguaje. Lo que implica realizar una distanciación temporal, una distanciación debida al canal y/o una distanciación instrumental. Concluimos argumentando que para ello es necesario que los decidores de programas de formación docente comprendan que una cosa es prescribir la formación de un profesional reflexivo y otra muy diferente el disponer de los recursos y las herramientas adecuadas para cumplir ese objetivo.

Palabras Clave: profesores, formación inicial, reflexión, política y estrategias.
\end{abstract}

\begin{abstract}
The present article covers three of our papers on critical thinking and the methodological approaches used in each one of them. It is a logical consequence of another one published by the same research team in 2010, revealing a critical view upon critical thinking. To begin with, this text stablishes the concept of critical thinking in order to, then, present each particular research, as well as to show the methodological difficulties undergone in order to confront the process and the process of critical thinking. The description of the research and the analytical dialogue developed by the team allow to stablish that the observation of critical thinking in an initial learning context needs manifestations of the order of actions or language. This implies temporal aliening due to the cannel or instrument. Conclusions allow arguing that it is necessary that those deciding upon planning and contents understand that one thing is proposing the critical initial formation and another one is to have the resources and tools necessary to accomplish that goal.
\end{abstract}

Key Words: teachers, initial formation, critical thinking, policy and strategies.

\section{RESUMO}

Faz-se a convergência de três trabalhos nossos referentes ao conceito de reflexão e abordagens metodológicas que temos dado a eles. Constitui-se sequência de outro trabalho publicado pelos mesmos autores em 2010, onde eles apresentam uma visão crítica de reflexão. Primeiro, no presente trabalho, apresenta-se resumidamente a nossa visão do conceito para, depois, situar nossas investigações e destacar a relevância dos desafios metodológicos 
que enfrentamos para estudar o processo de reflexão e o processo de desenvolvimento da reflexão. A descrição dos nossos estudos e o exercício do diálogo reflexivo que realizamos nos permitem sinalizar que a observação de reflexão no contexto da formação inicial requer a utilização de manifestações que podem ser da ordem da ação ou da linguagem. Isto implica um afastamento temporal, um distanciamento devido ao canal e/ou distanciamento instrumental. Conclui-se argumentando que isso exige que aqueles que decidem sobre os programas de formação de professores compreendam que uma coisa é prescrever a formação de um profissional reflexivo e outra bem diferente é dispor os recursos e as ferramentas adequadas para alcançar o que é programado por eles.

Palavras-chave: professores, formação inicial, reflexão, políticas e estratégias.

\section{LA REFLEXIÓN: UNA VISIÓN PANORÁMICA DEL CONCEPTO}

Considerada como garantía de profesionalización, la reflexión ocupa, después de largo tiempo, un lugar importante en el área de la formación docente. Es por ello que el discurso de organismos gubernamentales como el Consejo Superior de Educación (Conseil supérieur de l'éducation, 1991), en Quebec, apuestan a favor de la reflexión al señalar que ésta estimula la profesionalización de un oficio caracterizado por la interacción. En esa misma línea, el Ministerio de educación de Quebec (Ministère de l'Éducation [MEQ], 2001) impone un referencial de 12 competencias profesionales como marco de la formación de los futuros docentes de la provincia. Una de esas competencias pone a la reflexión como elemento clave en desarrollo profesional de la persona. Así, todo futuro docente es sensibilizado a la responsabilidad de desarrollarse profesionalmente por medio de la reflexión sobre su práctica.

Si bien el concepto de reflexión es ampliamente utilizado en el discurso y la documentación oficial, diversos investigadores relevan la complejidad de ese concepto, porque la reflexión seguiría un proceso de desarrollo propio a cada individuo y, en el contexto de la formación inicial docente, ésta evolucionaría durante un largo periodo de tiempo (Calderhead y Gates, 1993; Pultorak, 1996). La complejidad del concepto de reflexión se acentuaría al tomar en consideración la polisemia que la caracteriza, pues al ser utilizada en diversos campos de estudio como la sociología, la filosofía y la educación, entre otros, y al presentarla bajo diversas categorías como niveles de reflexión (SparksLanger et al., 1990; Van Manen, 1977), dimensiones de reflexión (Louden, 1991), tipos de reflexión (Valli, 1997) o perspectivas de reflexión (Grimmett et al., 1990), resulta normal que este concepto tome diferentes significados y que por lo mismo se produzca la confusión conceptual que le es reprochada (Beauchamp, 2006; Correa Molina et al., 2010; Desjardins, 2000; Ecclestone, 1996; Fendler, 2003; Grimmett et al., 1990; Saussez y Allal, 2007).

El análisis teórico de las definiciones de reflexión presentes en la literatura llevó a Beauchamp (2006) a determinar que, según los escritos analizados, el concepto de reflexión puede referirse a aspectos cognitivos, metacognitivos o lingüísticos; puede también corresponder a un proceso de resolución de problema o a un proceso de crítica social. El trabajo del autor revela siete lógicas subyacentes a este concepto, yendo desde el solo hecho de pensar distinto o con mayor claridad, al de realizar un proceso de transformación como persona para así transformar la sociedad.

Entre este abanico de concepciones, nuestro interés se focaliza en la actualización de la reflexión en los programas de formación docente. De manera más puntual, en el desarrollo que se supone debe producirse en los futuros profesionales durante su formación 
inicial, específicamente en el contexto de las prácticas. Es importante señalar que más que adherir a categorizaciones como las anteriormente mencionadas, hemos más bien privilegiado una visión de la reflexión como proceso intra e inter-psíquico, no lineal, por lo tanto no jerárquico, que participa en el desarrollo profesional de los futuros docentes (Correa Molina et al., 2010). En ese sentido, en contextos de formación inicial docente la reflexión se desarrollaría por medio de un proceso realizado en dos niveles: primero como un proceso de movilización, de adaptación y de renovación de recursos personales y profesionales de la persona; Segundo como un proceso de desarrollo articulado en torno a dos movimientos complementarios y progresivos, es decir la sistematización y la complejización de la reflexión en contextos de ejercicio profesional. De esta manera, los estudiantes en práctica sistematizarían su reflexión sobre aspectos de su práctica y complejizarían el abanico de recursos y de objetos de reflexión (Correa Molina et al. 2010) ${ }^{1}$. Bajo ese ángulo, el desarrollo de la reflexión en contexto de formación inicial se traduce en términos de una sistematización y complejización progresiva a medida del avance de la formación del estudiante (Correa Molina et al., 2010).

Además, si bien la reflexión y su desarrollo como procesos ocurren a nivel intrapersonal, éstos pueden ser estimulados por otra persona (profesor tutor, supervisor, un par, el grupo, etc.). Sin embargo, en contexto de formación y de investigación esos procesos deben ser externalizados, ser observables. Una manifestación posible de esos procesos, a fin de aprehenderles, es recurrir a la verbalización, ya que la manifestación por medio del lenguaje verbal, en tanto reconstrucción del proceso reflexivo efectuado precedentemente, permitiría aprehender la reflexión de manera precisa y sistemática; no obstante, el hecho de recurrir al lenguaje verbal implica necesariamente una distanciación del proceso de reflexión que él reconstituye (Correa Molina et al., 2010). La distanciación de la que hablamos en nuestros estudios es tanto temporal como instrumental: temporal porque la reflexión ocurre después de la experiencia, e instrumental porque, desde el punto de vista metodológico, hemos recurrido a medios como el registro video. Esos aspectos son presentados en las secciones que siguen.

\section{DESAFÍOS METODOLÓGICOS}

Interesarse por el estudio de la reflexión conduce ineluctablemente a la cuestión del método a utilizar. Primero, hay que considerar que tratándose de un proceso interno no podemos acceder a otra cosa que a la observación de sus manifestaciones, y eso con absoluta reserva. En efecto, según lo expuesto en la sección precedente, podemos constatar que su manifestación es dependiente de los instrumentos utilizados para provocarla y del interés o motivación de la persona a compartir su reflexión. Al recurrir al análisis del discurso verbal o escrito de aquel que comparte su reflexión, dependemos de su voluntad. No obstante esos límites, la reflexión suscita el interés de investigadores que buscan establecer dispositivos metodológicos susceptibles de aprehenderla. En esta sección presentamos tres proyectos de investigación que se dieron como objetivo estudiar la reflexión de futuros profesionales inscritos en programas de formación inicial. En las líneas que siguen presentamos esos estudios y ponemos en relieve los desafíos metodológicos enfrentados.

Esta visión es ampliamente detallada y documentada en el artículo en cuestión. 


\subsection{UN ESTUDIO QUE BUSCA «APREHENDER» LA REFLEXIÓN Y APUNTA AL MEJORAMIENTO} PROFESIONAL

Chaubet (2010) se pregunta si un entrenamiento a la reflexión en contexto de formación inicial es susceptible de desarrollar mecanismos durables de auto-renovación profesional. Su investigación identifica en primer lugar un conjunto de autores contemporáneos en práctica reflexiva ligados al pensamiento educativo, humanista y pragmático de Dewey (1933/2004, 1997, 2008), que inspiró el trabajo de Schön (1983) sobre el profesional reflexivo. Una definición operacional de la reflexión es así sintetizada y sirve de base al estudio de Chaubet para determinar dónde y en qué condiciones la reflexión se produce al interior de formaciones o proyectos que anuncian su ambición de hacer reflexionar. Las condiciones de emergencia así relevadas podrían poseer características reproductibles, posibles de reinvertir en formaciones de tipo reflexiva existentes o crearlas. El estudio apunta también a documentar formas genéricas de cuestionamiento, índices posibles de esquemas de construcción o de transformación profesional. En el fondo, se trata de poner en relieve un método para analizar los programas de formación, universitarios o no, para promover la reflexión.

La metodología del estudio releva varios desafíos ligados a la definición de la reflexión adoptada: un objeto triple comportando un proceso, un resultado y efectos psicológicos o físicos. El proceso cíclico sería desencadenado por una interpelación, objeto de perplejidad, incomodidad o simple curiosidad (Dewey, 1933/2004), en breve, un tipo de ruptura (Donnay y Charlier, 2006) en el flujo de la experiencia individual (Saussez y Allal, 2007). El actor, en formación o experimentado, buscaría naturalmente preservar la continuidad de ese flujo por una investigación deliberada y lógica, lógica derivada de la experiencia y no de un principio racional establecido a priori (Dewey, 2008). Esta búsqueda no estaría aislada en una burbuja intra-psíquica, ella se ubicaría bajo el signo de la interacción con el medio físico y con otras personas (Dewey, 1933/2004, 1997; Schön, 1983, 1987; St-Arnaud, 1992, 2001). Más importante aún, para las implicaciones metodológicas que nos preocupan aquí, esta búsqueda desembocaría en un resultado para el actor: un cambio de perspectiva, una comprensión renovada de la situación, en síntesis, una reconceptualización (próxima del concepto de recategorización de Clot, 2001) punto de apoyo de eventuales nuevas acciones (Bolton, 2005; Brockbank y McGill, 2007; Donnay y Charlier, 2006; Korthagen et al., 2001; Loughran, 2006; Moon, 2004; Osterman y Kottkamp, 2004).

Emociones y sentimientos juegan un rol motriz, incluso desencadenador en ese proceso, por lo tanto, su resultado no es sólo un producto cognitivo (Brockbank y McGill, 2007). Korthagen y Vasalos $(2005,2009)$ incluso afirman, para los futuros docentes, que en su reflexión ellos deben saber reconectarse con sus cualidades personales y motivaciones profundas. Sin embargo, no sería sino hacia el final del ciclo de investigación que el componente afectivo se manifestaría con mayor énfasis: efectos de energización o de empoderamiento (Donnay y Charlier, 2006; Osterman y Kottkamp, 2004), de sentimiento de dominio, de conexión con el otro y con el mundo, de pertenencia a una comunidad, etc. (Brockbank y McGill, 2007; Senge et al., 2004).

En síntesis, tendríamos aquí tres elementos: procesos, resultados y efectos, que pueden a la vez definir pragmáticamente la reflexión y constituir "herramientas metodológicas" para captarla. 


\subsubsection{Desafíos metodológicos}

Ningún dispositivo fue concebido específicamente en esta investigación para captar en vivo los eventos de reflexión colectiva o individual. Debimos fiarnos a los "instrumentos" testigos de la formación y del proyecto profesional estudiados, es decir las personas, y pedirles recordar momentos de su formación o de su trabajo. El investigador apostó por escuchar "el punto de vista del actor", que en definitiva es el que ocupa la mejor posición para hablar de lo que él vive y siente (Poupart et al., 1997). La gestión del proceso es así cualitativa y comprensiva, más que explicativa, y sigue el postulado epistemológico que señala que "toda persona puede entrar en la vivencia y sensación de otra persona" (Paillé y Mucchielli, 2008: 29) a condición de realizar el esfuerzo de crear un clima de escucha y empatía. Para extraer los eventos de reflexión de su sitio ecológico de producción y pasarlos bajo la lupa del investigador, Chaubet optó por la co-creación de indicios verbales (datos suscitados por Van der Maren, 2004), recogidos durante las entrevistas semi-estructuradas registradas y luego transcritas, es decir observables y accesibles. Una primera entrevista, colectiva, fue seguida por otra individual con cada participante para afinar la calidad de los datos recolectados.

Por otra parte, a fin de concentrar la mayor cantidad posible de manifestaciones de reflexión en el discurso, las entrevistas giraron alrededor de los temas siguientes: eventos sorprendentes o estimulantes, cambios de percepción experimentados durante la formación o el trabajo. Se suponía que: 1) los actores recuerdan más fácilmente los eventos que los interpelan o que modifican su forma de ver las cosas; 2) que varios de esos eventos podrían desde ya constituirse en desencadenadores, resultados o efectos de ocurrencia de reflexión, es decir ponerse directamente en el campo de observación definido por el investigador.

Varias distanciaciones metodológicas se intercalaron entre los eventos de reflexión y sus manifestaciones observadas: a) distancia espacio-temporal respecto a los lugares y momentos reales de desencadenamiento de la reflexión, o de los cambios de perspectiva o de los efectos físicos o psicológicos sobre sí; b) distancia debida a la inserción de un medio creado para ello (la entrevista); c) finalmente, una doble traducción lingüística: primero una verbalización-fijación de pensamientos sutiles y volátiles que quizás se produjeron en el momento bajo formas no verbales; enseguida, una cadena oral y una cadena escrita (verbatim) fiel, pero a las que le falta el tono, la entonación, la emoción de la voz, sin contar las mímicas, expresiones faciales, posturas corporales, gestos, etc. (Cosnier y Brossard, 1984). La pérdida de información no verbal durante el proceso de transcripción es, sin embargo, ligeramente compensada: el investigador se constituyó en instrumento de investigación al realizar él mismo las entrevistas (él guarda en su memoria la Gestalt que le recuerda, en el momento de la lectura de las transcripciones, la atmósfera general de tal o tal otra entrevista). Sin embargo, es posible preguntarse si los eventos de reflexión recogidos en las palabras de los participantes podrían ser tan dependientes del contexto de la muestra interrogada, que la operacionalización de la triple definición de la reflexión vendría a operacionalizar un modelo local, inaplicable a otros contextos. Para eliminar esa duda, tres muestras fueron constituidas en tres sitios: dos de ellos ligados a contextos diferentes de formación universitaria (enseñanza de francés como segundo lenguaje y cooperación internacional), y un tercero ligado a un contexto estrictamente profesional (incluso si éste se encuentra también en contexto universitario y relacionado con el programa de francés). De esta manera, el investigador pudo concentrarse en las características de la "pinza metodológica" destinada a captar las "ocurrencias de reflexión”. 


\subsubsection{Límites del estudio}

Los participantes voluntarios de las tres muestras podrían tener un perfil de reflexión particular susceptible de orientar el estudio. El hecho de que elementos vividos precisos, objetivos, aparezcan en el discurso de unos y otros atenúa en cierta medida este límite.

Por otra parte, el aporte estructurador del investigador y su voluntad de aprehender los eventos de reflexión pueden también, paradojalmente, constituir un obstáculo metodológico. El enfoque deductivo, concretamente la pauta conceptual aplicada a cada verbatim, podría minimizar los horizontes de descubrimiento como lo expresa Giorgi (1997: 355) “(...) con estrategias de investigación de este tipo, uno no encuentra otra cosa que lo que se había previsto al comienzo". El instrumento metodológico experimentado estaría destinado a tener éxito. Un enfoque inductivo, en emergencia fenomenológica, es entonces simultáneo al trabajo, a dos niveles de lectura de las transcripciones: el de la observación de las manifestaciones de reflexión del actor (¿tal pasaje que yo leo entra en la definición de la reflexión adoptada?), y más allá, el del valor heurístico de la definición de la reflexión utilizada para observar (¿esta triple definición es operacional?). Es la cuestión de los límites de la "pinza" utilizada: sus fortalezas y territorios prohibidos, su pertinencia respecto a las situaciones observadas y a las zonas grises, su fineza de captación, los objetos que debe dejar de lado, etc.

Otra interrogante refiere a la capacidad de esta pinza para captar no sólo una reflexión intra-psíquica "simple", sino la polinización cruzada de pensamientos surgidos de las interacciones sociales en el medio profesional o de formación, de las maneras de hacer, de decir o no decir o de la influencia intangible del entorno cultural (Clot, 1999; Bruner, 1986/2008). ¿Podemos hablar de franco límite entre un mundo "interior" (nuestro pensamiento) y un mundo social "exterior" cuando finalmente lo social, en una concepción vygotskiana, se encontraría tanto al interior como al exterior? (Clot, 2008). ¿Hasta dónde un instrumento metodológico construido en torno a un ciclo reflexivo intra-psíquico puede dar cuenta de la influencia del "otro" en la reflexión individual?.

Finalmente, lo observado es sólo una instantánea tomada a través de un lente (entrevistas de 90 minutos), reducido por el poder de evocación de la memoria de los actores, por su capacidad para encontrar las palabras justas para expresarse (Eraut, 2000) y a la del investigador para interpretar sin traicionar el discurso de los actores, todo ello por medio de un obligado "desvío semántico" (Demazière y Dubar, 2004). En resumen, la reflexión aquí abordada es esencialmente reconstituida de fragmentos dispersos e incompletos, de indicios o huellas. Hablamos entonces de reflexión "pasada", aunque comprendemos que ella puede influenciar el futuro. Por otro lado, aunque la reflexión emergente durante la entrevista no es el objeto de estudio, no podemos negar que ésta podría desempeñar un papel en la reconstitución/reconstrucción mnemónica de la reflexión pasada.

\subsection{UN ESTUDIO QUE BUSCA «CAPTAR» LA PRÁCTICA REFLEXIVA DE ESTUDIANTES EN PRÁCTICA POR MEDIO DE LA INTERACCIÓN EN LÍNEA.}

En complementariedad al enfoque de Chaubet (2010), Collin (2009) se interesa por la dimensión colectiva de la reflexión en formación inicial docente, específicamente en la interacción en línea como apoyo a la práctica reflexiva de los estudiantes en práctica. Su estudio se basa en la idea de que los medios de comunicación por Internet parecen propicios 
a sostener la práctica reflexiva gracias a su potencial socio-cognitivo (Depover et al., 2007; Gunawardena, et al., 1997, Jonassen, 2000). Además, éstos revelan ser particularmente pertinentes durante las prácticas ya que los estudiantes se encuentran dispersos en diferentes lugares, lo cual reduce las ocasiones de interacción en presencia.

Sin embargo, la asociación de la interacción en línea (como la interacción frente a frente) y de la práctica reflexiva, a primera vista muy pertinente a nivel práctico, no está formalizada a nivel teórico, porque la práctica reflexiva se conceptualiza generalmente como un proceso psíquico individual (Ottesen, 2007; Zeichner y Liston, 1996) y no colectivo, incluso si ciertos autores reconcilian cada vez más estos dos polos al ponerlos en un continuo. Este estudio se focaliza también en discutir, a nivel teórico, la asociación de la práctica reflexiva como fenómeno intra-personal, y de la interacción verbal como fenómeno inter-personal. Al respecto, la teoría de la mediación semiótica (Vygotsky, 1962) aporta una mirada interesante a la práctica reflexiva en situación de interacción verbal, sea ésta frente a frente o en línea. Esta teoría sugiere que el desarrollo de las funciones mentales superiores de un individuo es el resultado de la internalización de sus interacciones sociales, siendo la interacción verbal el medio principal. Al asociar la práctica reflexiva de Schön (1983) y la mediación semiótica de Vygotsky (1962), este estudio propone el marco conceptual de la práctica reflexiva interactiva, que sugiere que la reflexión de los docentes puede ser estimulada particularmente a través de sus interacciones verbales sobre su práctica docente, antes que ellos la internalicen para poder explotarla de manera autónoma en su docencia. Este marco conceptual considera la interacción verbal como un "motor" y un indicador de la práctica reflexiva de los docentes. Éste se aplica únicamente a contextos donde los docentes discuten entre ellos sobre sus experiencias profesionales, lo que implica necesariamente que no se encuentren impartiendo docencia. Es decir, se trata de una reflexión sobre la acción (Schön, 1983). En cambio, es inválido cuando el docente está en acción (impartiendo docencia), porque al encontrarse aislado de sus colegas ninguna interacción entre ellos es posible. Consecuentemente, en el caso de la práctica reflexiva docente, este marco conceptual cubre la reflexión sobre la acción y no la reflexión en acción (Schön, 1983).

Los diferentes contextos de la práctica reflexiva, normados por el número (individual versus colectivo) y la relación con la acción (durante o después de la acción), condicionan el hecho de que la práctica reflexiva interactiva corresponda a la que se da en un contexto de reflexión colectiva posterior a la acción.

\subsubsection{Desafíos metodológicos}

La manifestación discursiva de la práctica reflexiva en las interacciones de los actores educativos es la cuestión central de este estudio. Se trata de preguntarse cómo observar los "indicios" de práctica reflexiva en la interacción verbal de los docentes sobre su práctica y cómo la distanciación debida al canal utilizado (oral o escrito) afecta esos indicios. Para aprehender ese desafío metodológico, este estudio se basa en un análisis de interacciones en línea asincrónicas generadas por futuros docentes en el marco de su última práctica. Las interacciones en línea provienen de tres grupos de estudiantes en práctica (unos doce por grupo) que tienen la instrucción de utilizar una lista de difusión para discutir sobre su experiencia docente durante su última práctica. Se trata entonces de un dispositivo de acompañamiento que adiciona una distanciación instrumental de orden pedagógico a 
la distanciación debida al canal antes mencionado. Las interacciones en línea recogidas durante la práctica representan datos invocados, que se consideran como garantía de una cierta autenticidad de los indicios discursivos de la práctica reflexiva interactiva, tal cual es manifestada en ese contexto específico de formación. Así, es por un análisis de las interacciones en línea de estudiantes en práctica, como espacio de co-construcción de la reflexión (Gunarwardena, et al., 1997), que este estudio intenta circunscribir la construcción de la práctica reflexiva de futuros docentes durante su formación inicial. El análisis de las interacciones en línea es acompañado de entrevistas individuales y grupales y de un cuestionario. Los objetivos de este estudio son (1) examinar cómo se construye la práctica reflexiva durante las interacciones en línea; (2) determinar el papel de la interacción en línea (particularmente frente a otros dispositivos) para el desarrollo de la práctica reflexiva de los estudiantes en práctica.

\subsubsection{Límites del estudio}

Varios límites deben ser mencionados para situar con precisión los aspectos cubiertos por este estudio. Comenzamos por precisar que el estudio de la práctica reflexiva interactiva es aprehendida a nivel empírico de manera doblemente parcial: (1) en primer lugar, éste se limita a la interacción en línea y, por lo mismo, no permite informar la práctica reflexiva interactiva generada en una interacción frente a frente; (2) enseguida, este estudio examina la interacción en línea por medio de una lista de difusión integrada en las modalidades de las prácticas de la Universidad de Montreal. De este modo, este medio de comunicación por Internet no puede pretender representar el rico y polimorfo espectro de la interacción en línea y, por ello, no puede tampoco representar los diversos dispositivos que estimulan la práctica reflexiva de manera colectiva en los diferentes programas de formación inicial docente.

Debemos recalcar que las interacciones verbales recolectadas en este estudio, difieren de aquellas que pueden encontrarse habitualmente en el medio profesional docente por varias razones: primero, se trata de interacciones verbales de docentes en proceso de formación inicial, y no de docentes en función; además, la interacción verbal tratada aquí es escrita y no oral. Las interacciones verbales de los docentes sobre su práctica profesional son habitualmente orales (discusiones informales en la sala de profesores, reuniones de equipo, etc.); finalmente, la participación de los estudiantes en práctica en la lista de difusión es evaluada en el marco de su práctica, lo que puede ser considerado como un límite en la medida que las interacciones en línea recolectadas en esta investigación tienen un valor académico y obligatorio más que profesional y voluntario. Por sus características, es entonces posible pensar que las interacciones en línea recolectadas aquí no son representativas de las interacciones verbales (en línea o frente a frente) producidas por docentes en función, lo que significa que los resultados obtenidos tienen una transferibilidad limitada.

Este estudio cubre de manera parcial la práctica reflexiva interactiva. Así, en estudios subsecuentes sería interesante multiplicar los actores (ejemplo: estudiantes en práctica, docentes de experiencia, supervisores, etc.) y los tipos de interacciones (frente a frente, en línea sincrónica, en línea asincrónica), a fin de identificar con mayor precisión las manifestaciones discursivas de la práctica reflexiva interactiva y determinar cuáles contextos de interacción serían más propicios para sostenerla y estimularla. 


\subsection{UN ESTUDIO QUE BUSCA CAPTAR EL DESARROLLO DE LA COMPETENCIA REFLEXIVA}

Correa Molina y Gervais $(2009,2012)$ se interesan en descubrir indicios de la progresión del desarrollo de la reflexión en contexto de formación inicial docente. Para responder a una de las 12 competencias profesionales del referencial que rige la formación docente en Quebec (MEQ, 2001), los futuros docentes deben demostrar su desarrollo profesional al manifestar su capacidad de reflexionar sobre su práctica. Desde ese punto de vista, la reflexión es percibida como un recurso que permitiría a los futuros profesionales de la docencia el participar activamente en su propio progreso. La postura adoptada en este estudio se aproxima de lo constatado por Gervais et al. $(2007,2008)$ que sitúan la reflexión como una competencia transversal que jugaría un papel importante en el desarrollo de otras competencias. Vista así, la reflexión sería más bien una metacompetencia, porque permitiría orquestar los recursos de la persona para que ésta pueda actuar eficazmente.

De manera más específica, Correa Molina y Gervais privilegian el contexto de las prácticas porque, durante la formación, ellas constituyen el único contexto auténtico de movilización de recursos y de manifestación de competencias para actuar de manera eficaz y adecuada en el ejercicio de la profesión. En efecto, a medida de su formación, los futuros docentes deben manifestar las competencias prescritas por el referencial que el ministerio impuso para la formación docente en Quebec (MEQ, 2001). Es importante mencionar que la noción de competencia vehiculada por el referencial es la del conjunto de recursos, internos y externos, que permite al profesional actuar de manera eficaz en un contexto específico (Le Boterf, 2002). Este actuar circunstanciado implica, por una parte, la presencia de un abanico de recursos y, por otra parte, la capacidad y posibilidad de movilizar esos recursos para responder adecuadamente a la situación (Le Boterf, 2002; Tardif, 2006).

Al buscar trazar una trayectoria de desarrollo de la reflexión, este estudio se sitúa en una perspectiva de análisis cualitativo descriptivo. Schön (1983), inspirado por los trabajos de Dewey, deja entrever que reflexionar implica un cierto diálogo reflexivo con la situación en donde el lenguaje da acceso al repertorio de experiencias del individuo, lo que le permitiría relevar una nueva estructuración de la situación. Si la reflexión implica un diálogo consigo mismo o con otro a propósito de una situación cualquiera, nos parece pertinente afirmar que para acceder a la manifestación de la reflexión de los estudiantes en práctica, hay que cederles la palabra. ¿Qué mejor que sea el mismo actor quién explicite el sentido de su acción? Por otro lado, la presencia de un "otro", que interpela y tranquiliza a la vez, contribuiría a la explicitación de la acción y a la reflexión sobre la acción.

El enfoque de la argumentación práctica de Fenstermacher (1996) nos sirvió para apoyar la reflexión de los estudiantes en práctica. Este enfoque está en coherencia con una concepción amplia de las dimensiones a examinar en la reflexión sobre la práctica. Según Fenstermacher (1996), diversas premisas de acción pueden ser verbalizadas por un docente para explicitar lo que fundamenta su actuar: conocimientos teóricos, saber de experiencia, valores, elementos contextuales. Esto refleja una concepción de la reflexión que toma en cuenta diferentes contenidos sobre los cuales puede referirse la reflexión como, por ejemplo, el modelo "cebolla" propuesto por Korthagen y Vasalos (2009) que comprende el entorno del docente, sus comportamientos, competencias, creencias, identidad profesional y sentido de su misión. Si bien el enfoque de Fenstermacher fue retenido para los efectos de este estudio, debemos precisar que originalmente el autor trabajó con docentes de experiencia que acompañó durante varios meses. Hicimos entonces una adaptación del 
enfoque para poder utilizarlo en el contexto de las prácticas que, evidentemente, ocurre en tiempos limitados y con personas que están en proceso de formación y que, en consecuencia, no poseen aún la experiencia de ser docentes a cabalidad.

\subsubsection{Desafíos metodológicos}

Querer analizar la práctica profesional a fin de conocer lo que fundamenta la acción profesional debe tomar en cuenta el sentido que el actor da sus actos. De acuerdo a lo anterior, este último debe ser considerado como autor y primer analista de su acción. Esto impone un primer y no menor desafío metodológico, porque para acceder a los fundamentos de la acción profesional es necesario crear un clima de confianza para que el actor ocupe el rol principal en ese proceso en que la interacción es privilegiada y en donde él está llamado a tomar la palabra. Es por eso que los trabajos de Fenstermacher $(1994,1996)$ nos parecieron adecuados ya que consideran dos estrategias que cuadran bien con el marco de nuestro estudio: el trabajo en colegialidad, es decir la práctica reflexiva interactiva, y el recurso a la videoscopía como forma de distanciación instrumental. Este enfoque se realiza en dos fases: explicitación y reconstrucción. Durante la fase de explicitación, el docente ayudado de un "otro" (investigador o par) es llevado a explicitar los fundamentos de su acción. Enseguida, a fin de juzgar de su conveniencia o exactitud, sus argumentos son analizados en función de criterios éticos, empíricos y otros, para que así estos puedan constituir una base válida para la acción. Por razones materiales y ecológicas (respeto del contexto de las prácticas de los estudiantes), sólo la primera fase fue utilizada. Por otra parte, contrariamente al enfoque original, aquí es el actor y no el investigador el que decide los elementos de su práctica que él desea analizar. Observar su práctica y la de sus pares, reflexionar en grupo sobre su práctica y la de un camarada puede, en cierta medida, contribuir a modificar ciertos aspectos de la práctica sin que exista intención explícita de parte del investigador. Estos aspectos, entre otros, constituyen la adaptación del enfoque de Fenstermacher (Correa Molina y Gervais, 2010) del que hablábamos en la sección precedente.

El papel que juega el "otro" es de importancia capital, porque es él el que debe crear el necesario clima de respeto para suscitar el cuestionamiento sin caer en el enjuiciamiento de la acción observada. La interacción entre los estudiantes en práctica participantes y el investigador, durante los encuentros colectivos de explicitación de la práctica, provoca la emergencia de datos suscitados. Son los mismos estudiantes en práctica los que eligieron el segmento del registro video de una de sus clases, filmada hacia el final de su periodo de práctica, para presentarlo al investigador y sus pares durante el encuentro de reflexión compartida. El dejar que sean los propios estudiantes quienes elijan el material de análisis persigue ciertamente ponerlos en confianza en el exigente proceso de reflexión en presencia de otras personas, pero igualmente responsabilizarlos en cuanto a elegir lo que es susceptible de desencadenar realmente en ellos, en ese momento de su trayectoria formativa, un proceso de reflexión. Sin embargo, existe el riesgo de que la elección del segmento sea hecha en función de criterios de evitación para, por ejemplo, librarse de situaciones de su práctica que resultarían inconfortables de visionar en compañía de los pares y del investigador, pero que tendrían un gran potencial como desencadenadores de reflexión.

Además de los análisis colectivos de la práctica, se llevaron también a cabo entrevistas semi-dirigidas individuales con los profesores colaboradores y los supervisores de los estudiantes en práctica participantes del estudio. El material que servía como base de esas 
entrevistas consistía en la presentación del segmento video seleccionado por el estudiante en práctica. El cruce de informaciones provenientes de la discusión entre los estudiantes en práctica y el investigador, basada en los indicios de su práctica (registros video), así como la entrevista con sus formadores (profesores colaboradores y supervisores), pretende aproximarse a datos auténticos.

\subsubsection{Límites del estudio}

Aunque el enfoque metodológico presenta ventajas, también presenta límites. Al respecto, mencionemos que los participantes son todos voluntarios, lo que representa un límite porque podemos suponer que se trata de individuos que ya poseen una cierta tendencia a reflexionar sobre sus acciones, a asumir una postura de análisis de su práctica. Personas con la suficiente confianza en sí mismas para exponerse frente a otras personas con sus fortalezas y límites a fin de dotarse de medios para mejorar su acción profesional. Por lo tanto, los resultados del estudio no pueden generalizarse a los futuros docentes en su conjunto.

Por otra parte, la presencia del investigador durante los encuentros de explicitación puede conducir a los participantes a intentar adivinar y responder las supuestas expectativas de éste, lo cual representa un límite importante en cuanto al valor de los datos así recolectados. Estudiar el desarrollo de la reflexión en ese contexto equivale quizás a suscitar un desarrollo, sin saber si ese mismo proceso sucedería sin la intervención del investigador.

\section{DISCUSIÓN Y CONCLUSIÓN}

El objetivo de este texto era el de poner en relieve los desafíos metodológicos que implica el estudio del desarrollo o de las manifestaciones de la reflexión en contexto de formación profesional. Consecuentemente, adoptamos una postura analítica del aspecto metodológico de los estudios aquí expuestos para recalcar los desafíos y límites de los métodos privilegiados en cada uno de ellos. En razón de ello y de los límites de espacio, optamos por no presentar resultados de cada uno de estos estudios.

Recordemos que la reflexión es un concepto difícil de circunscribir, particularmente por el hecho de ser aprehendido por diferentes visiones cuyas posturas teóricas no son siempre equivalentes o compatibles las unas con las otras. Además, independientemente de esta situación, recordemos también que en el contexto de la formación inicial docente el paradigma del profesional reflexivo es omnipresente. Lo cual no significa que discurso y operacionalización de ese discurso cohabiten en armonía al interior de los programas de formación. Creemos entonces que el primer paso es el de tomar consciencia de esos aspectos y, en segundo lugar, ver cómo disminuir la brecha entre lo que se profesa y lo que realmente se realiza al respecto en los programas de formación docente. Puesto que ponemos en relieve las fortalezas y límites de las metodologías utilizadas en nuestros estudios, creemos que este artículo puede contribuir a alimentar la necesaria "reflexión" para que los programas trabajen en esa armonización.

En este texto precisamos las dimensiones ecológicas y metodológicas del proceso de reflexión en formación profesional, de manera particular en formación inicial docente. En ese sentido, distinguimos dos procesos ampliamente imbricados en formación inicial: el proceso de reflexión y el proceso de desarrollo de la reflexión. Definimos este último 
como un movimiento progresivo de sistematización y complejidad de la reflexión durante la formación inicial y por un contexto profesional específico. La conceptualización a nivel intra-psíquico de estos dos procesos implica que su observación con fines pedagógicos o científicos pasa necesariamente por el intermedio de manifestaciones que pueden ser del orden de la acción o del lenguaje. En el segundo caso, la observación de la reflexión por el lenguaje verbal puede hacer intervenir tres tipos de distanciación: una distanciación temporal, una distanciación debida al canal tomado y una distanciación instrumental. Esta última puede ser de naturaleza pedagógica y permite generar indicios de reflexión que los formadores pueden explotar para evaluar la reflexión de sus estudiantes. Esos indicios pueden igualmente ser utilizados a fines científicos, constituyendo en ese caso datos invocados que el investigador recolecta después de ser producidos. Paralelamente a la distanciación instrumental pedagógica, las investigaciones sobre la reflexión inducen generalmente una distanciación instrumental por intermedio de entrevistas individuales o grupales, encuentros de explicitación, de reflexión compartida, etc. En ese caso, esos instrumentos generan datos suscitados. La Figura 1 permite representar los elementos recién mencionados.

Figura 1. Dimensiones metodológicas del proceso de reflexión (traducido de Correa Molina et al., 2010).

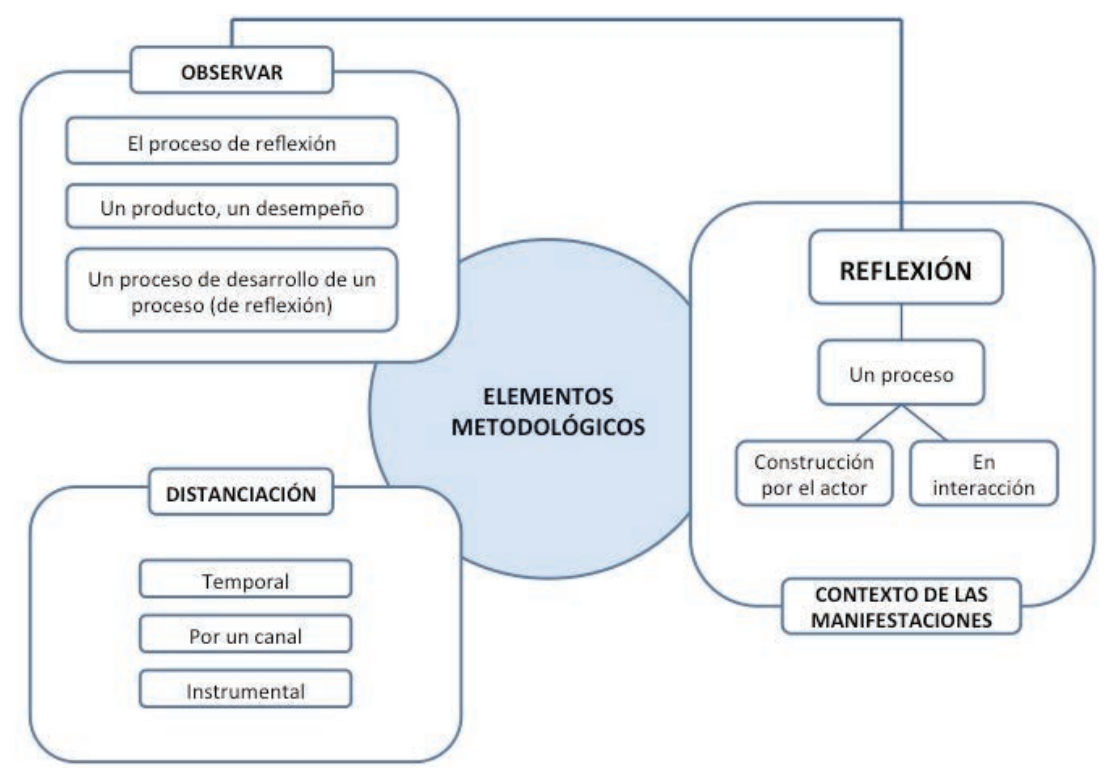

El esquema presentado en la Figura 1 resume las discusiones y reflexiones colectivas que sostuvimos regularmente mientras llevábamos a cabo estos tres estudios: tesis en el caso de Chaubet y de Collin y proyecto subvencionado en el caso de Correa Molina y Gervais. Este esquema permite no sólo situar nuestros estudios respectivos en cuanto a su complementariedad y a su especificidad, sino que también en cuanto a que propone una cartografía de la formación y del estudio de la reflexión en contexto de formación inicial. Lo cual puede ser interesante para abrir nuevas perspectivas de investigación. Los desafíos metodológicos relevados en los tres trabajos de investigación presentados, revelan 
la importancia de la distanciación en el estudio tanto del proceso de reflexión como en el del proceso de desarrollo de la misma. En consecuencia, cuestionan a la vez la manera de captar los indicios de reflexión. Si eso es materia de preocupación para el respeto del rigor de los protocolos de investigación, debiera serlo también para los programas de formación y los dispositivos supuestamente destinados a estimular el desarrollo de la reflexión de futuros profesionales. Al respecto, frente a la polifonía de voces que cantan elogios sobre la reflexión, necesario es preguntarse ¿cómo asegurar en los formadores una apropiación conceptual armónica de un concepto tan polisémico y polémico como el de reflexión?

Al respecto, creemos que es necesario que los programas de formación docente den respuesta a esa interrogante. En la actualidad, a pesar de las buenas intenciones declaradas, los programas no logran cumplir con las metas anunciadas en el discurso de formar profesionales reflexivos. Esto, porque: a) las herramientas utilizadas para promover la reflexión generalmente no superan los niveles de descripción de hechos (Correa Molina y Gervais, 2009) y, b) la voluntad no va necesariamente en coherencia con la disponibilidad de recursos humanos y financieros para el logro de una formación docente de calidad que permita que, primero, en contexto de formación inicial, los futuros docentes sean sensibilizados y formados al análisis reflexivo y que, segundo, en contexto de ejercicio profesional éstos cuenten con los tiempos y condiciones necesarias al mantenimiento de la sistematización de la reflexión trabajada durante sus estudios universitarios como sello de profesionalidad. Sin condiciones laborales adecuadas, ni el mejor de los programas de formación logrará que los docentes mantengan los aprendizajes adquiridos en su formación inicial sobre la sistematización del proceso reflexivo, susceptible del continuo mejoramiento de la práctica profesional docente.

Finalmente, a pesar que en este artículo no se hayan presentado resultados por cada uno de los estudios descritos, igual estamos en posición de señalar algunos efectos o repercusiones a dos niveles distintos y complementarios: a nivel de la definición de la reflexión y de su contenido y a nivel de las modalidades de desarrollo de la reflexión. Al respecto, ya señalamos que, desde nuestra perspectiva, la reflexión se sitúa al nivel de metacompetencia y no de competencia. Estas repercusiones permitirían al fin tocar diferentes facetas de la reflexión en formación inicial docente para perfeccionar su inclusión en ese campo. Así, una de las primeras repercusiones se relaciona con la definición misma del concepto de "competencia reflexiva" y la dificultad actual de circunscribirla y delimitar su contenido, ya que es relativamente vaga. Una primera finalidad de la investigación es entonces contribuir a clarificar y comprender mejor ese concepto. En seguida, como las modalidades de desarrollo de la reflexión en formación inicial docente son muy variables de un programa a otro y las condiciones de eficacia de los dispositivos utilizados para ello deben ser mejor documentados, la segunda finalidad de la investigación debería ser la de contribuir a operacionalizar el proceso de reflexión y, consecuentemente, el desarrollo del mismo en contexto de formación inicial.

\section{REFERENCIAS BIBLIOGRÁFICAS}

Beauchamp, C. (2006). Understanding Reflection in Teaching: A Framework for Analyzing the Literature (Tesis doctoral). McGill University, Montreal, Canadá.

Bolton, G. (2005). Writing and Professional Development ( $2^{\text {nd }}$ Edition). London, Thousand 
Oaks, New Delhi: Sage Publications.

Brockbank, A., \& McGill, I. (2007). Facilitating Reflective Learning in Higher Education (2 Edition). Maidenhead, Berkshire: Open University Press, McGraw-Hill Education, McGraw-Hill House.

Bruner, J. (2008). Culture et modes de pensée. Paris: Retz. (Trabajo original publicado en 1986). Chaubet, P. (2010). Saisir la réflexion pour mieux former à une pratique réflexive: d'un modèle théorique à son opérationnalisation. Éducation et Francophonie, vol.38, n.2, 60-77.

Clot, Y. (1999). La fonction psychologique du travail. Paris: PUF.

Clot, Y. (2001). Clinique du travail et problème de la conscience. Travailler, n.6, 31-54.

Clot, Y. (2008). Travail et pouvoir d'agir. Paris: PUF.

Calderhead, J., \& Gates, P. (1993). Conceptualizing Reflection in Teacher Development. London: The Falmer Press.

Collin, S. (2009). La pratique réflexive interactionnelle: rapport entre la pratique réflexive et l'interaction verbale. Revue canadienne des jeunes chercheur(e)s en éducation, vol.2, n.1, 1-9.

Conseil supérieur de l'éducation (1991). Rapport annuel 1990-1991 sur l'état et les besoins de l'éducation. La profession enseignantte: vers un renouvellement du contrat social. Québec: Les Publications du Québec.

Correa Molina, E., Collin, S., Chaubet, P., et Gervais, C. (2010). Concept de réflexion: un regard critique. Éducation et Francophonie, vol.38, n.2, 135-154.

Correa Molina, E., et Gervais, C. (2012). Contenus alimentant la réflexion de stagiaires en début de formation. En M. Tardif, C. Borges et A. Malo (Eds.) Le virage réflexif en éducation. Où en sommes nous 30 ans après Schön? (pp. 195-213). Bruxelles: DeBoeck.

Correa Molina, E., et Gervais, C. (2010). Une adaptation de l'approche de Fenstermacher pour explorer la communication du savoir d'expérience. En F. Yvon et F. Saussez (Eds.). Analyser l'activité enseignante: des outils méthodologiques et théoriques pour l'intervention et la formation. (pp. 229-250). Québec: Presses de l'Université Laval.

Correa Molina, E., \& Gervais, C. (2009). Reflective Competency: How can it be developed in practicum? Paper $13^{\text {th }}$ Biennal Conference of the European Association for Research on Learning and Instruction (EARLI), Amsterdam, Pays-Bas.

Cosnier, J., et Brossard, A. (1984). La communication non verbale. Delachaux et Nietslé: Neuchâtel.

Demazière, D., et Dubar, C. (2004). Analyser les entretiens biographiques: l'exemple de récits d'insertion. Saint-Nicolas: Les Presses de l'Université Laval.

Depover, C., Karsenti, T., et Komis, V. (2007). Enseigner avec les technologies. Québec: Presses de l'Université du Québec.

Desjardins, J. (2000). Analyse critique du champ conceptuel de la formation réflexive (Tesis doctoral). Université de Montréal, Montreal, Canadá.

Dewey, J. (2008). Dewey, the Later Works, 1925-1953 - Volume 12: 1938 - Logic: The Theory of Inquiry. Carbondale: Southern Illinois University Press.

Dewey, J. (2004). Comment nous pensons. Paris: Les Empêcheurs de penser en rond. (Trabajo original publicado en 1933)

Dewey, J. (1997). Experience and Education. New York: Touchstone.

Donnay, J., et Charlier, E. (2006). Apprendre par l'analyse de pratiques: Initiation au compagnonnage réflexif. Namur: Presses Universitaires de Namur, Sherbrooke: Éditions du CRP.

Ecclestone, K. (1996). The reflective practitioner: mantra for a model for emancipation? Studies in the Education of Adults, vol.28, n.2, 146-161.

Eraut, M. (2000). Non-formal learning and tacit knowledge in professional work. British Journal of Educational Psychology, vol.70, n.1, 113-136.

Fendler, L. (2003). Teacher reflection in a hall of mirrors: historical influences and political reverberations. Educational Researcher, vol.32, n.3, 16-25. 
Fenstermacher, G. (1996). Les arguments pratiques dans la transformation morale de l'enseignement d'une discipline. Revue des sciences de l'éducation, vol.22, n.3, 617-634.

Fenstermacher, G., et Richardson, V. (1994). L'explicitation et la reconstruction des arguments pratiques dans l'enseignement. Cahiers de la recherche en éducation, vol.1, n.1, 157-182.

Gervais, C., Correa Molina, E., et Lepage, M. (2008). Comment se construisent les compétences liées à l'acte d'enseigner? Explicitation de pratiques pendant les stages. En E. Correa Molina et C. Gervais (Eds.), Les stages en formation à l'enseignement: pratiques et perspectives théoriques (pp. 153-175). Québec: Presses de l'Université du Québec.

Gervais, C., Correa Molina, E., \& Lepage, M. (2007). Teaching Competencies during Pre-service Training: What do we Know about their Construction? Communication au Symposium Studying Teacher Education du SIG Teacher Education, 12 Conférence biennale de l'EARLI, Budapest.

Giorgi. A. (1997). De la méthode phénoménologique utilisée comme mode de recherche qualitative en sciences humaines: théorie, pratique et évaluation. En J. Poupart, J.-P. Deslauriers, L.-H. Groulx, A. Laperrière, R. Mayer et A.P. Pires, (Eds.), La recherche qualitative, enjeux épistémologiques et méthodologiques (pp. 341-364). Montréal: Gaëtan Morin Editeur.

Grimmett, P., Erickson, G., Mackinnon, G., \& Riecken, T. (1990). Reflective practice in teacher education. In R. Clift, R. Houston, \& M. Pugach (Eds.), Encouraging Reflective Practice in Education: An Analysis of Issues and Programs (pp. 20-38). New York: Teachers College Press.

Gunawardena, C., Lowe, C., \& Anderson, T. (1997). Analysis of a global online debate and the development of an interaction analysis model for examining social construction of knowledge in computer conferencing. Journal of educational computing research, vol.17, n.4, 397-432.

Jonassen, D.H. (2000). Computers as mindtools for schools: engaging critical thinking. Upper Saddle River, New Jersey: Prentice Hall.

Korthagen, F., Kessels, J., Koster, B., Langerwarf, B., \& Wubbels, T. (2001). Linking Practice and Theory: The Pedagogy of Realistic Teacher Education. Mahwah, New Jersey: Lawrence Erlbaum Associates, Publishers.

Korthagen, F., \& Vasalos, A. (2005). Levels in reflection: core reflection as a means to enhance professional growth. Teachers and Teaching, vol.11, n.1, 47-71.

Korthagen, F., \& Vasalos, A. (2009). From Reflection to Presence and Mindfulness: 30 years of developments concerning the concept of reflection in teacher education. $13^{\text {th }}$ Biennal Conference of the European Association for Research on Learning and Instruction (EARLI), Amsterdam, Pays-Bas.

Le Boterf, G. (2002). Développer la compétence des professionnels ( $4^{\mathrm{e}}$ édition revue et mise à jour de Compétence et navigation professionnelle). Paris: Éditions d'Organisation.

Louden, W. (1991). Understanding Teaching: Continuity and Change in Teachers' Knowledge. New York: Teachers College Press Columbia University.

Loughran, J.J. (2006). Developing a Pedagogy of Teacher Education: Understanding teaching and learning about teaching. London and New-York: Routledge.

Ministère de l'Éducation (2001). La formation à l'enseignement: les orientations, les compétences professionnelles. Québec: Gouvernement du Québec.

Moon, J. (2004). A Handbook of Reflective and Experiential Learning: Theory and Practice. London and New York: RoutledgeFalmer.

Osterman, K., \& Kottkamp, R. (2004). Reflective Practice for Educators: Professional Development to Improve Student Learning ( $2^{\text {nd }}$ Edition). Thousand Oaks: Corwin Press.

Ottesen, E. (2007). Reflection in teacher education. Reflective Practice, vol.8, n.1, 31-46.

Paillé, P., et Mucchielli, A. (2008). L'analyse qualitative en sciences humaines et sociales. Paris: Armand Colin.

Poupart, J., Deslauriers, J.-P., Groulx, L.-H., Laperrière, A., Mayer, R., et Pires, A.P. (1997). La recherche qualitative, enjeux épistémologiques et méthodologiques. Montréal: Gaëtan Morin Editeur.

Pultorak, E. (1996). Following the developmental process of reflection in novice teachers: Three years of investigation. Journal of Teacher Education, vol.47, n.4, 283-291. 
Saint-Arnaud, Y. (1992). Connaître par l'action? Montréal: Les presses de l'Université de Montréal.

Saint-Arnaud, Y. (2001). La réflexion-dans-l'action: un changement de paradigme. Recherche et formation, n.36, 17-27.

Saussez, F., et Allal, L. (2007). Réfléchir sur sa pratique: le rôle de l'autoévaluation? Mesure et évaluation en éducation, vol.30, n.1, 97-124.

Schön, D.A. (1983). The reflective practitioner. New-York: Basic Book.

Schön. D.A. (1987). Educating the Reflective Practitioner: towards a new design for teaching and learning professions. San Francisco: Jossey-Bass.

Senge, P., Scharmer, C.O., Jaworski, J., \& Flowers, B.S. (2004). Presence: Exploring Profound Change in People, Organizations, and Society. New York: Doubleday.

Sparks-Langer, G., Simmons, J., Pash, M., Colton, A., \& Starko, A. (1990). Reflective pedagogical thinking: How can we promote it and measure it? Journal of Teacher Education, vol.41, n.5, 23-32.

Tardif, J. (2006). L'évaluation des compétences. Documenter le parcours de développement. Montréal: Chenelière Éducation.

Valli, L. (1997). Listening to other voices: a description of teacher reflection in the United States. Peabody Journal of Education, vol.72, n.1, 67-88.

Van Der Maren, J-M. (2004). Méthodes de recherche pour l'éducation. Bruxelles, De Boeck

Van Manen, M. (1977). Linking ways of knowing with ways of being practical. Curriculum Inquiry, vol.6, n.3, 205-228.

Vygotsky, L. S. (1962). Thought and language. Cambridge: M.I.T. Press.

Zeichner, K., \& Liston, D. (1996). Reflective teaching: an introduction. Mahwah: Lawrence Erlbaum Associates. 